Voix et Images

voixetimages

\title{
Devant quelques photos de famille
}

\section{Michel Biron}

Volume 26, numéro 1 (76), automne 2000

\section{L'immonde}

URI : https://id.erudit.org/iderudit/201526ar

DOI : https://doi.org/10.7202/201526ar

Aller au sommaire du numéro

\section{Éditeur(s)}

Université du Québec à Montréal

\section{ISSN}

0318-9201 (imprimé)

1705-933X (numérique)

Découvrir la revue

\section{Citer cet article}

Biron, M. (2000). Devant quelques photos de famille. Voix et Images, 26(1),

177-182. https://doi.org/10.7202/201526ar d'utilisation que vous pouvez consulter en ligne.

https://apropos.erudit.org/fr/usagers/politique-dutilisation/ 


\section{Devant quelques photos de famille}

\section{Michel Biron, Université du Québec à Montréal}

Depuis La note de passage ${ }^{1}$, publié en 1985, François Gravel publie régulièrement des romans agréables, marqués d'un certain humour contemporain, des romans qui appartiennent à ce que la sociologie de la littérature appelle l'art moyen. Il le fait mieux que d'autres, même si ses succès sont plus modestes que ceux de plusieurs de ses confrères. Le plus récent roman d'Yves Beauchemin, Les émois d'un marchand de café ${ }^{2}$, ne vaut pourtant pas le dernier-né de Gravel, Fillion et frères ${ }^{3}$. Après
Ostende et Miss Septembre ${ }^{4}$, dont les personnages étaient de plus en plus minces, on pouvait, il est vrai, se demander s'il n'allait pas, lui aussi, se contenter de reprendre les mêmes procédés narratifs, s'il n'allait pas tout simplement rédiger ses romans au lieu de les écrire. Ce n'est pas une mince affaire de donner le goût au lecteur de tourner la page, roman après roman. De très bons auteurs, comme Francine Noël avec La conjuration des bâtards ${ }^{5}$, n'y arrivent pas toujours. D'autres n'y parviennent 
jamais tout à fait, comme ce pauvre François Barcelo qui se plaint d'écrire des best-sellers qui ne se vendent pas. Ce n'est donc pas rien de constater que Fillion et frères possède déjà cette qualité: il se laisse lire.

Le roman de Gravel raconte les aventures d'une famille montréalaise depuis la Crise de 1929 jusque dans les années 1970. Ils sont huit enfants, dont trois créeront, après s'être initiés de diverses manières aux lois de la concurrence, une lucrative entreprise canadienne-française de meubles, "Fillion et frères". Leur success story constitue la trame du roman, son moteur, peut-être même aussi une sorte d'allégorie du roman en tant qu'entreprise artisanale qui ne méprise pas les vérités du commerce, étant lui aussi un commerce, c'est-à-dire tout à la fois un dialogue et un échange intéressé avec un lecteur qui ne demande pas mieux que de jouer le jeu. Le véritable jeu se déroule toutefois en deçà et au-delà de cette histoire banale, hommage aujourd'hui répandu au génie de Québec Inc. Le récit de cette entreprise intéresse moins que les portraits des trois frères et du reste de la famille. L'histoire ne nous est d'ailleurs pas racontée par un narrateur extérieur à ce monde, mais bien par le fils de Louis, l'un des trois propriétaires du magasin de meubles. Du passé, il ne connaît que les grandes lignes et les petites anecdotes, les souvenirs de famille.

Ces souvenirs épars s'organisent d'abord autour d'événements connus: la Crise, qui jette le grand-père à la rue et qui force les garçons à s'inventer un petit commerce pour subvenir aux besoins immédiats de la famille; la guerre, d'où le fils aîné écrit des lettres que sa mère lira à haute voix à l'église devant toute la paroisse réunie. Puis la toile de fond sociale s'efface pour ne laisser que les secrets de famille plus ou moins gardés, comme ceux que partage la société de l'Ordre de Jacques-Cartier (appelée vulgairement La Patente) à laquelle Louis adhère sans enthousiasme, rien que pour frayer avec les banquiers canadiens-français dont il espérait la collaboration, pour faire contrepoids aux marchands juifs. $\mathrm{Ce}$ n'est pas qu'il soit franchement nationaliste ou xénophobe, mais il se laisse porter par le mouvement général, par l'époque. De toute façon, il ne croit pas à grand-chose, cet homme terne: ni aux vertus du commerce, ni à celles de la famille. Devenu vendeur malgré lui, doué pour un métier qui ne lui dit rien, il ne fait pas un personnage extrêmement intéressant. Pourtant, le portrait de ce "grand maigre", extraordinairement timide (il ne s'aventure du côté des femmes qu'à l'âge de vingt-cinq ans), occupe une bonne partie du roman et reçoit, dans les toutes dernières pages, un éclairage inattendu. Une ancienne prostituée, inconnue de la famille, se présente au salon funéraire pour rendre un hommage inquiétant à cet homme que tout le monde croyait sans histoire.

Le roman est ainsi fait qu'aucun personnage ne peut prétendre au statut de héros: c'est la famille qui règne ici. Non pas le clan structuré autour d'un père qui domine, mais une famille convenablement fraternelle, dont le père est d'emblée écarté, suivi de peu par le frère aîné. Les figures les plus autoritaires sont ici celles de la mère, Annette, et de la fille aînée, surnommée la Reine Margot. 
Personne ne comprend d'où celle-ci tire son pouvoir, mais le fait est là : malgré sa laideur indiscutable, elle en impose à tous, y compris à ses quatre maris qui meurent tour à tour après lui avoir consenti tout ce qu'elle voulait. De leur. côté, les frères ne se mêlent guère des affaires d'autrui et n'ont d'autre satisfaction que celle de voir leur nom sur l'enseigne du magasin, "Fillion et frères". Édouard, passé du cours classique (il se destinait à la prêtrise) au commerce, est un excellent vendeur quand il n'est pas en train de boire. Louis n'est pas présenté comme un alcoolique, mais il boit presque autant que son frère, avec davantage de discrétion, cachant des petits "mickey " en divers endroits. Seul Léo, le moins doué des trois en apparence, garde une distance salutaire par rapport au commerce. De temps en temps, il laisse échapper un vrai mot d'esprit qui mystifie et enrage ses frères; c'est aussi le seul personnage heureux, constate le narrateur.

Fillion et frères est un roman léger qu'on serait mal venu de lire autrement, en exigeant de lui qu'il soit ce qu'il ne prétend pas être. Mais un roman dit toujours un peu plus (ou un peu moins) que ce qu'il croit dire. En l'occurrence, il arrange à sa manière le récit de l'ascension sociale d'une famille canadienne-française au milieu du $\mathrm{xx}^{\mathrm{e}}$ siècle. Il lui fait subir une curieuse opération en cours de route. Lentement, cette histoire s'amincit, se retourne sur elle-même et s'assèche, coupée de ce qui la nourrissait au départ. Au début du roman, nous sommes à Montréal dans une famille tout ce qu'il y a de plus typique, mais cela n'empêche pas les personnages d'appartenir à un en- semble plus vaste: la Crise, la guerre, Montréal. Puis le monde se referme sur la seule famille et rien d'autre n'existe que ce "nous". Or, ce repli s'effectue précisément au moment où Louis décide de quitter son patron juif, un certain Lunn, que le narrateur appelait Monsieur l'Homme. À l'inverse des frères Fillion, ce personnage énigmatique, élégant, cet artiste aux mains de pianiste ne semble appartenir à aucune famille, pas même à celle des Juifs montréalais même si on dit "le Juif " pour parler de lui : contrairement au stéréotype, c'est un piètre marchand installé (on ne sait trop pourquoi) loin du quartier juif, un dandy qui saisit mal le sens local du commerce. En revanche, "Louis comprend d'instinct l'âme du client canadien-français ${ }^{6}$. Ce dernier aurait $\mathrm{pu}$ continuer à travailler auprès de son patron, mais sa mère le convainc, grâce au seul argument raciste ("Il est Juif, c'est tout. Ça veut tout dire. Ne me fais pas parler pour rien $\left.{ }^{7} . . \cdots\right)$, de fonder son propre magasin, de travailler à une nouvelle enseigne, celle de la famille. Montréal n'est plus alors une ville d'Amérique, portée par l'Histoire du siècle, mais un espaçe forclos, coupé du reste du monde, coupé de sa propre réalité sociale et culturelle. Il n'y a plus ni Juifs, ni Irlandais ni même une société vivante et complexe: il n'y a que Fillion et frères.

"Avec Fillion et frères, lit-on en quatrième de couverture, c'est une grande partie de notre histoire qui resurgit, attachante, drôle, renouvelée. "Je m'arrête un instant sur le pronom "notre" utilisé pour décrire cette histoire d'une famille canadiennefrançaise: "Notre histoire"? Qui se cache derrière ce "nous" que 
l'éditeur emploie comme si de rien n'était, comme s'il allait de soi que le lecteur allait se reconnaître dans cette collectivité "naturellen. Se peut-il que le roman ne s'adresse qu'à ce lecteurlà, à un "nous "fait d'avance? Le "nous" de la quatrième de couverture ressemble dangereusement à cette famille si complètement repliée sur elle-même qu'elle en oublie le monde extérieur. D'ailleurs, la deuxième partie du roman verse dans une sorte de régionalisme abstrait, avec des échos obligés aux débats idéologiques de l'heure, par exemple à l'émancipation des femmes. Je cherche en vain l'intérêt romanesque d'un chapitre comme celui où Louis se demande si sa femme Cécile a le droit de travailler: c'est de la pure reconstitution idéologique. Nous ne sommes plus dans le roman lui-même, mais dans une présentation de clichés qui n'intéresse même pas ceux qui font partie de la photo.

Ce régionalisme abstrait n'a pas de thèse proprement dite à défendre, à la façon du roman du terroir. Il propose encore moins une utopie à la manière de celles qu'on trouvait dans des romans du XIX ${ }^{\mathrm{e}}$ siècle. Même entre la famille et le monde, les liens sont absents, alors qu'ils étaient nombreux et nécessaires chez des auteurs de chronique familiale comme Michel Tremblay ou Roger Martin du Gard. Entre Louis et le narrateur, son fils, le fossé n'en est pas seulement un de génération. Père et fils n'entretiennent aucun conflit, donc aucun lien. Les seuls liens qui tiennent sont fondés sur la contemporanéité dans laquelle tout ressemble à tout et à rien. Il n'y a pas de filiation véritable, il n'y a qu'une affiliation de frères sans grande affi- nité par ailleurs. Cette famille soidisant typique appartient à un monde attachant, peut-être, mais surtout irréel, dans lequel d'ailleurs le lecteur se reconnaît de moins en moins à mesure que le récit se rapproche de l'époque actuelle. À la fin, le seul passé qui puisse exister dans ce roman faussement historique est le passé contemporain, c'est-à-dire un passé entièrement soumis aux règles du présent, de la familiarité absolue. Les lois de succession historique n'opèrent plus qu'en fonction d'une identité posée comme préalable. Cette identité est cependant la plus fragile qui soit: dès que surgit un étranger, il se produit alors une impression d'irréalité comparable à celle que suscite la rencontre, au salon funéraire, d'une inconnue, anciennement prostituée, qui fut, mais comment est-il possible que vous ne l'ayez jamais su, l'amie la plus intime de votre propre père. Comme quoi le "nous" d'aujourd'hui fuit de toutes parts, même lorsqu'il est censé s'enrouler autour de ce bâton ancien, cette troisième jambe qu'on appelait naguère l'histoire.

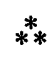

Qui se souvient de Claire Martin? Elle a publié l'an dernier un court recueil de nouvelles, Toute la vie ${ }^{8}$, après vingt-cinq ans de silence. Ce recueil comprend des textes écrits dans les années soixante ainsi que des inédits beaucoup plus récents. Peu d'écrivains québécois savent exprimer les émotions les plus indéterminées avec une écriture aussi précise et ferme que Claire Martin. Peu possèdent à un tel degré la maîtrise de la langue, peu s'amusent avec au- 
tant de facilité apparente avec les rouages de la narration, peu cherchent moins qu'elle à impressionner le lecteur. C'est discret, c'est léger, c'est même souvent drôle. À cause de quelques nouvelles moins réussies, au ton volontairement nostalgique, on pourrait croire qu'il s'agit d'une œuvre inactuelle, typique d'une autre génération. Mais allez lire "Conversation le soir dans une auberge de campagne " ou "La petite fille lit " et vous verrez que nous ne sommes pas loin de la Gabrielle Roy de Rue Deschambault.

On se convaincra un peu plus d'avoir sous-estimé Claire Martin en lisant son dernier roman, tout à fait inédit celui-là, intitulé L'amour impuni ${ }^{9}$. Ce court roman, paru lui aussi à L'instant même, ressemble à une longue nouvelle. Un homme observe une photographie de lui-même alors qu'il avait six ans. Il se voit, se revoit tel qu'il fut, semblable et méconnaissable. Puis il regarde les autres photographies enfouies dans la même boîte, celles de sa mère, de son père et de sa sœur ainée. Cela suffit pour lui donner le goût d'écrire: "j'écris ma réflexion sur ces photos qui me troublent ${ }^{10_{n}}$. Il écrit donc sur des photos de famille, sur le trouble que les photos suscitent, images du passé le plus proche, le plus intime. Pourquoi ce trouble? Cela fait partie des quelques questions sans réponse que le narrateur traîne avec lui, "comme ça ${ }^{11}$ ".

Si L'amour impuni se lit comme une nouvelle, c'est que tout le texte tourne autour d'un seul thème, évoqué dès le titre, l'amour homosexuel. Michel Tremblay, dans La nuit des princes charmants ${ }^{12}$, avait abordé le même sujet en insistant surtout sur le dépucelage du héros. L'amour im- puni ne raconte pas une initiation sexuelle, mais une histoire d'amour aussi simple que possible, heureuse par surcroît, entre le narrateur et un de ses étudiants en géologie, Philippe. Le milieu familial du narrateur, il faut dire, est tout à fait différent de celui que décrit Tremblay: ses parents accueillent spontanément leur gendre avec une affection sincère, presque avec fierté. Ce sont pourtant des gens assez traditionnels, style vieille France. Mais ils croient à l'amour plus qu'à n'importe quoi et ils se réjouissent de savoir leur fils capable d'aimer. Tout va donc bien. Cela ne suffit pas toutefois à lever le trouble que ce dernier a ressenti devant les photographies. Son amour impuni se gagne contre quelque chose, il ne va pas entièrement de soi. D'où les rituels un peu solennels des commencements, qu'ils suivent avec un zèle presque suspect. Lors de leur voyage à Niagara (les clichés amoureux se donnent libre cours quand les amants croient échapper au péché d'insignifiance), ils s'enthousiasment de leur conjugalité naissante, partagent les gestes quotidiens, se pâment de bonheur. C'est sympathique, quoiqu'un peu ennuyeux à la longue, à tel point qu'on se met à regarder ailleurs, à jeter un coup d'œil aux autres photographies.

Il n'en faut pas plus pour s'apercevoir que les personnages du père et de la mère ont plus de couleur que la figure trop parfaite de Philippe. L'amour impuni, n'est-ce pas aussi celui que partage jusqu'à la toute fin ce couple faussement conventionnel? L'une des plus belles pages de ce roman (car c'en est bien un finalement) est celle du récit que fait le père de la mort de sa femme, 
ou plutôt non pas de la mort en ellemême, mais de l'étonnante cérémonie amoureuse qu'il accomplit devant le corps désormais inanimé de celle qu'il aime et désire plus que jamais. "Son récit m'a si complètement saisi, écrit le narrateur, qu'en entrant chez moi je l'ai écrit sans attendre, mot à mot, il me semble, et je l'ai relu souvent. J'ai pris, pour cela, mon plus beau papier, celui que j'utilise pour répondre aux "grandes invitations". C'est, en effet, la grande invitation à l'amour ${ }^{13}$." Curieusement, cette scène d'amour produit un trouble bien plus fort que l'amour homosexuel du narrateur. L'impudeur d'un père de famille parlant ainsi à son fils est infiniment moins attendue que les tendresses mièvres des deux jeunes amoureux. Voici un extrait de cette "grande invitation à l'amour ":

Je lui ai fermé les yeux que j'ai baisés après, puis la bouche qui était douce et fraîche. Avant de vous avertir et d'appeler le médecin, j'ai pris le temps de caresser son visage, ses bras, ses seins, ses pieds jolis, sa peau aussi douce que la nuit de nos noces. J'ai pris son sexe dans ma main, je l'ai pressé en murmurant "merci et j'ai pensé: merci pour m'avoir donné nos enfants. J'ai eu envie de couper une boucle de ses cheveux. J'y ai renoncé par crainte qu'un jour, on ne sait comment, ils soient jetés à la poussière du hasard. [...] Maintenant, je ne prendrai plus jamais personne dans mes bras comme je la tenais, elle. Jamais personne ne se blottira près de moi avec, comme elle disait, une "idée derrière la tête". Il me reste l'amour au cour, mais l'amour tangible, je ne connaîtrai plus jamais ça ${ }^{14}$.

Tel est le langage de Claire Martin: sous la simplicité du vocabulaire et de la syntaxe, quelque chọse d'extrêmement personnel surgit, le corps se met à parler presque directement, nous le voyons oser faire le geste le plus vrai, le plus grave, le plus humain. Pour reprendre une image biblique qu'affectionne Claire Martin, la prose du narrateur résonne tout à coup comme le "Cantique des cantiques".

1. François Gravel, La note de passage, Monréal, Boréal, 1985, 199 p.

2. Yves Beauchemin, Les émois d'un marchand de café, Montréal, Québec Amérique, 1999. $495 \mathrm{p}$.

3. François Gravel, Fillion et frères, Montréal, Québec Amérique, 2000, 347 p.

4. Id., Ostende, Montréal, Québec Amérique, 1994, 348 p.; Miss Septembre, Montréal, Québec Amérique, 1996, 223 p.

5. Francine Noël, La conjuration des bâtards, Montréal, Leméac, 1999, 513 p.

6. François Gravel, Fillion et frères, p. 76.

7. Ibid., p. 121.

8. Claire Martin, Toute la vie, Québec, L'instant même, 1999, $111 \mathrm{p}$.

9. Id., L'amour impuni, Québec, L'instant même, 2000, 145 p.

10. Ibid., p. 9.

11. Ibid., p. 9.

12. Michel Tremblay, La nuit des princes cbarmants, Montréal/Arles, Leméac/Actes Sud, 1995, $221 \mathrm{p}$.

13. lbid., p. 131.

14. Ibid., p. 132-133. 